\title{
Nanoscale
}

(D) Check for updates

Cite this: Nanoscale, 2018, 10, 14245

\section{The chemical fingerprint of hair melanosomes by infrared nano-spectroscopy $\dagger$}

\author{
Vesna Stanic, (ID *a Francisco Carlos Barbosa Maia, (D) a Raul de Oliveira Freitas, (ID) \\ Fabiano Emmanuel Montoro (iD ${ }^{b}$ and Kenneth Evans-Lutterodt (iD c
}

Received 17th April 2018, Accepted 30th June 2018 DOI: $10.1039 / \mathrm{c} 8 \mathrm{nr} 03146 \mathrm{k}$ rsc.li/nanoscale

\begin{abstract}
In situ characterization of the chemical and structural properties of black and white sheep hair was performed with a spatial resolution of $25 \mathrm{~nm}$ using infrared nano-spectroscopy. Comparing data sets from two types of hair allowed us to isolate the keratin FTIR fingerprint and so mark off chemical properties of the hair's melanosomes. From a polarization sensitive analysis of the nano-FTIR spectra, we showed that keratin intermediate filaments (IFs) present anisotropic molecular ordering. In stark contrast with white hair which does not contain melanosomes, in black hair, we spatially resolved single melanosomes and achieved unprecedented assignment of the vibrational modes of pheomelanin and eumelanin. The in situ experiment presented here avoids harsh chemical extractive methods used in previous studies. Our findings offer a basis for a better understanding of the keratin chemical and structural packing in different hair phenotypes as well as the involvement of melanosomes in hair color and biological functionality.
\end{abstract}

\section{Introduction}

The chemical and physical properties of the hair are mainly determined by the keratin intermediate filaments (IFs) and the protein matrix. However, the hair local structure is also strongly dependent on secondary components such as lipids and minerals, as well as pigmented melanosomes. ${ }^{1-8}$ In particular, the partition of these secondary components between the main hair regions (medulla, cortex and cuticle) and the subtle differences of IF orientations yield unique physical characteristics and complexity which are not completely understood. For example, the wavelength dependent differential absorption of light by the pigmented melanosomes results in the color of mammalian hair. ${ }^{9}$ In contrast, the color in bird's feathers and insect's wings is most often generated by the interaction of light with photonic nanostructured crystals. ${ }^{10-12}$ However, in some species of lizards and birds, it is defined by a combination of both phenomena. ${ }^{13-15}$

Melanosomes are specialized cell organelles which produce melanin or pigment ${ }^{16,17}$ and can be found in the hair, skin, eyes and brain. Also, it has been reported that melanosomes

\footnotetext{
${ }^{a}$ Brazilian Synchrotron Light Laboratory, CNPEM, Campinas, SP 13083-970, Brazil. E-mail:vesna.stanic@lnls.br; Tel: +55 1935121044

${ }^{b}$ Brazilian National Nanotechnology Laboratory, CNPEM, Campinas, SP 13083-970, Brazil

${ }^{c}$ National Synchrotron Light Source - II, Brookhaven National Laboratory, Upton, NY 11973, USA

$\dagger$ Electronic supplementary information (ESI) available. See DOI: 10.1039/ C8NR03146K
}

exist in four distinct stages of maturation. ${ }^{16,17}$ Pigment production and structural and chemical changes during melanogenesis in these four stages are highly complex and still not completely known. It is important to point out that hair contains mostly mature melanosomes of the fourth stage of maturation. ${ }^{18}$

Several methods have been proposed for the extraction of melanosomes ${ }^{19}$ from hair, but usually such chemical treatments of the hair alter the chemistry and structure of the extracted melanasomes. Recently, T. Gorniak et al. ${ }^{20}$ investigated iridial melanosomes from inbred strains of mice, reporting genotype-specific differences in their granularity and surface morphology. Furthermore, they found that melanosomes with different origins are constituted of different building blocks, leading to different final packing and sizes with the consequence of different bulk properties of different types of melanosomes. It is important to also mention that melanosomes have many complex biological functions, since they are correlated to different diseases like Alzheimer's, albinism and skin cancer. For that reason, it is critical to understand and connect the physical and chemical characteristics of melanosomes with their biological properties.

Here we will limit our discussion only to the black and white sheep hair. We use nano-resolved Fourier transform infrared (nano-FTIR) spectroscopy to depict and discriminate, for the first time, the native vibrational state of two kinds of melanosomes: pheomelanin and eumelanin, directly in hair and without any chemical treatment. We chiefly focus on keratin and on the two types of melanosomes: pheomelanin, which has a red-brown color, and eumelanin with brown-black 
color. These melanosomes possess distinct chemical and physical properties, e.g. color and response to oxidative stress.

In previous studies, Fourier transform infrared micro-spectroscopy ( $\mu$-FTIR) was used for studying the chemistry of hair under label-free conditions. ${ }^{21,22}$ Most of the hair components is IR active in the fingerprint region $\left(500 \mathrm{~cm}^{-1}\right.$ to $\left.1700 \mathrm{~cm}^{-1}\right)$ and in the $\mathrm{C}-\mathrm{H}$ stretching region related to lipids $\left(\sim 2900 \mathrm{~cm}^{-1}\right.$ or $\sim 3.4 \mu \mathrm{m}$ ). However, as a diffraction-limited technique (Abbe limit), $\mu$-FTIR was unable to access the sub-micron internal structures of the hair, such as the melanosomes of interest here, which are typically less than $0.5 \mu \mathrm{m}$ in size.

Scattering scanning near-field optical microscopy $(\mathrm{s}-\mathrm{SNOM})^{23-25}$ has emerged as a powerful tool for the study of nano-localized chemical domains. Initially developed to operate with narrow-band laser sources for nanoscale-resolved imaging, s-SNOM made broadband mid-IR nano-spectroscopy feasible when it is combined with broadband IR sources (nano-FTIR). ${ }^{26-31}$ As a novel spectroscopy modality, nano-FTIR has been a decisive tool in several multidisciplinary investigations such as nano-heterogeneity of polymer blends, ${ }^{32,33}$ nano-mapping of individual protein complexes, ${ }^{34}$ nano-chemistry of multi-phase bio-minerals ${ }^{35}$ and local physics of atomically-thin materials. ${ }^{36-40}$ Organic materials are still the most challenging systems to be investigated by nano-FTIR due to their low scattering power compared to polar crystals. In this scenario, a variety of new developments aim at the study of the sub-wavelength organic chemistry of materials such as local thermal expansion, ${ }^{41-45}$ peak-force IR microscopy, ${ }^{46}$ synchrotron AFM-IR ${ }^{47}$ and opto-nanomechanical spectroscopy. ${ }^{48}$ Most of these techniques are based on the local expansion of the materials; hence, the mechanical detection allows for higher sensitivity to organic vibrational resonances as well as provides a more direct interpretation of the data. On the other hand, the spatial resolution in thermal expansion microscopies is defined by the thermal diffusion of the beam power into the material, which is a complex and non-controlled phenomenon. In s-SNOM and nano-FTIR, the optical probe is only defined by the AFM tip radius; therefore, it is still the only technique able to perform ultra-broadband IR spectroscopy with $25 \mathrm{~nm}$ resolution.

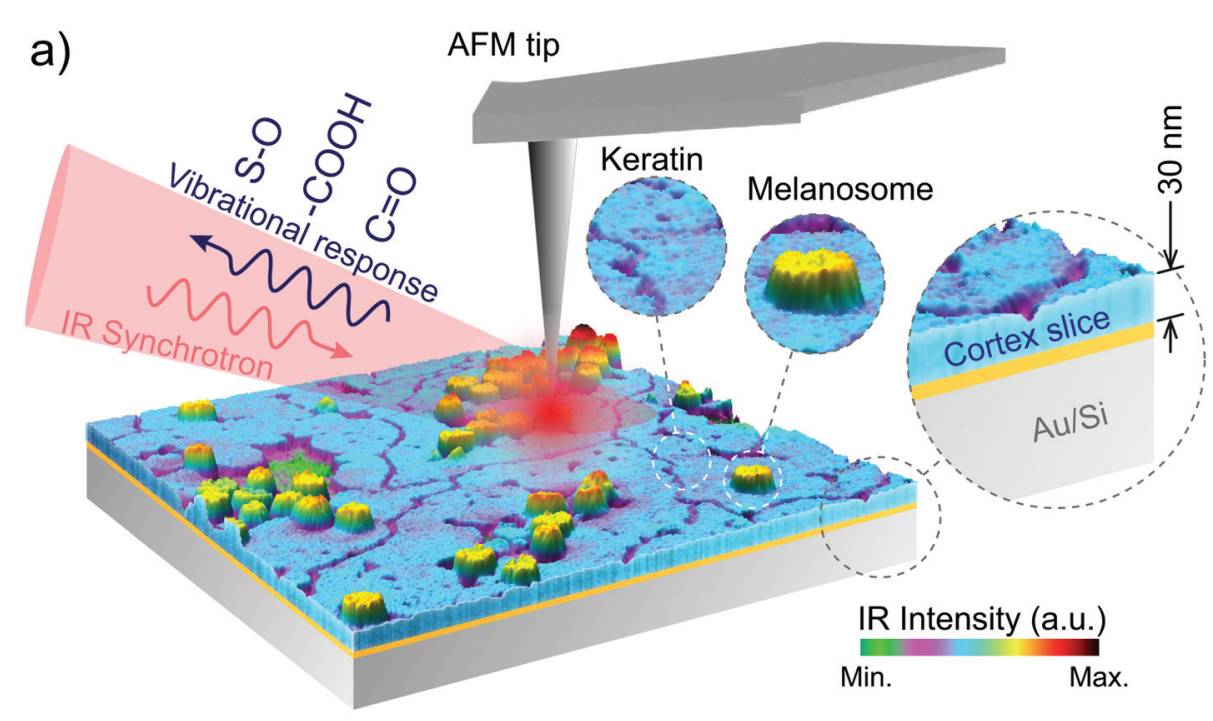

b)

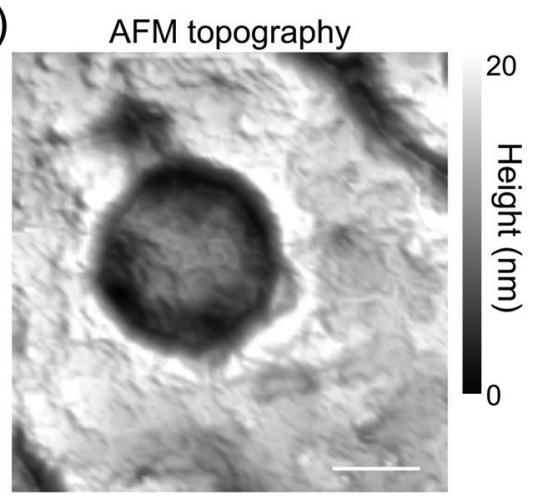

c)

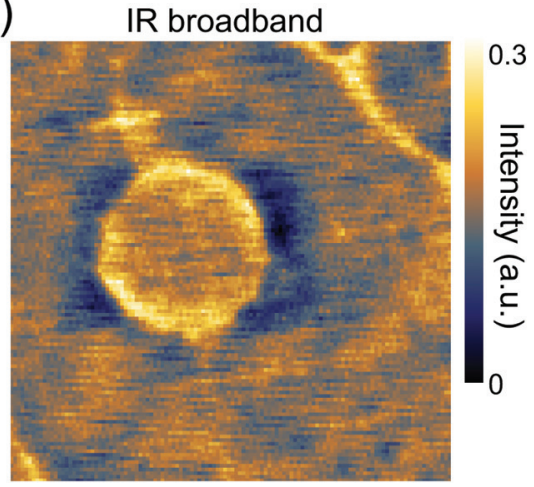

Fig. 1 Nano-FTIR experiment of a nano-slice of virgin sheep hair (a). As shown in (b) and (c) the AFM topographic and IR broadband images (1 $\times$ $1 \mu \mathrm{m}^{2}$ ) are obtained simultaneously and prior to the nano-FTIR spectra collection in order to select a precise location for measurements. As shown in (a) the synchrotron infrared light illuminates the scanning tip and also the sample. Near-field back-scattered light from the tip is filtered and processed according to s-SNOM interferometric procedures, resulting in nano-FTIR spectra for each investigated point on the sample. Spatial resolution is determined by the tip radius, which is of the order of $25 \mathrm{~nm}$ in this experiment. Scale bar in (b) represents $200 \mathrm{~nm}$. 
In this study, we apply nano-FTIR and scanning transmission electron microscopy (STEM) to study the morphological and chemical properties of virgin sheep black and white hair (for simplicity we will use the general term hair instead of wool). In the cortex region nano-FTIR unveils the chemical differences between keratin and melanosomes.

\section{Experimental}

\subsection{Sample preparation for nano-FTIR measurements}

Sheep hair or wool (European origin, Pramenka) was voluntarily donated by farmers and the agriculture department of the University of Zagreb, Croatia. The sheep hair was virgin, since it had never been treated with any reagents except normal washing with water. However, in spite of our efforts we should mention that sunlight, water or mechanical cutting of hair during slicing can induce some damage.

For nano-FTIR measurements we first embedded the hair in resin (Pelco Eponate 12 with BDMA) in order to cut slices of $30 \mathrm{~nm}$ thickness. Several hair strands were placed horizontally in a mold on a flat silicon wafer that keeps the hair centered in the middle of the mold and leveled. No stretching, pressure, or deformations were applied to the hair. After that, the resin was poured over the hair and allowed to cure at room temperature.
Before the nano-FTIR measurements, the hair was sliced to $\sim 30 \mathrm{~nm}$ thickness using an ultramicrotome (model: RMC Boeckeler PowerTome X). The slices were deposited on a clean gold substrate $(100 \mathrm{~nm}$ thick $\mathrm{Au}$ film sputtered on silicon wafer) as shown in Fig. 1 and dried at room temperature to evaporate the excess of water which is used in the ultramicrotome cut. Thus, on each Au substrate, we obtained several samples (hair cross-section and longitudinal slices).

\subsection{Nano-FTIR measurements}

Nano-FTIR was performed at the IR1 beamline of the Brazilian Synchrotron Light Laboratory. ${ }^{31}$ Based on s-SNOM, nano-FTIR uses broadband IR light as an excitation source to perform nano-scale spectroscopy. In our setup, the broadband IR radiation from the synchrotron illuminates a metallic AFM tip (Arrow-NCPt-W, Nanoworld) of a commercial s-SNOM microscope (NeaSNOM, Neaspec GmbH). ${ }^{31}$ In the semi-contact mode the tip oscillates in its natural resonant frequency $\Omega$. The IR beam induces charge separation and, accordingly, confinement of near-field radiation at the tip apex that turns into a nano-antenna, which has nearly an achromatic response to the IR excitation. As a result, the optical near-field of the tip polarizes an area on the sample given by the tip radius $(\sim 25 \mathrm{~nm})$. The light scattered from the effective polarization of the tip-sample system, carrying the modulation at frequency

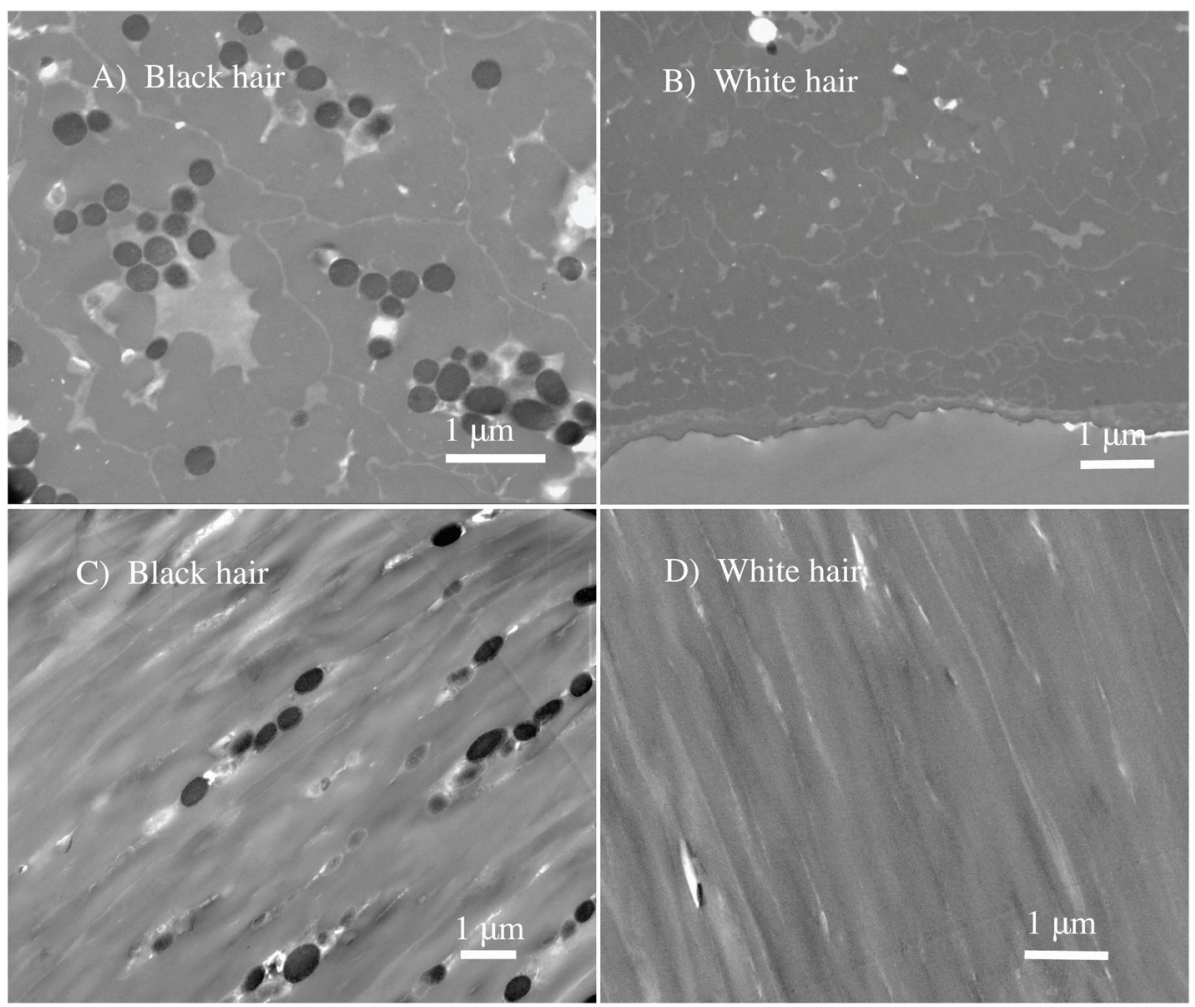

Fig. 2 STEM images of the black and white sheep hair. (A) The cross-section of the cortex of the black sheep hair showing the clustered black round melanosome organelles that are present only in black sheep hair. (B) The cross-section of the cortex of the white sheep hair showing the complete absence of melanosomes. (C) The longitudinal section of the cortex of the black sheep hair showing the black ellipsoidal melanosome organelles, clustered in arrays. (D) The longitudinal section of the cortex of the white sheep hair showing the oriented keratin macro-fibrils. 
$\Omega$, corresponds to the optical near-field scattering. We used a Mercury Cadmium Telluride detector (MCT KLD-0.1, Kolmar Technologies Inc.) coupled to a lock-in based detection scheme to acquire the background-free optical near-field by demodulation of the higher harmonics of $\Omega .{ }^{49}$ To attain nano-FTIR, the scattered near-field is directed to an asymmetric Michelson interferometer that, by the Fourier transform of the interferogram, produces the IR point-spectrum with the spatial resolution given by the tip apex. In our experiments, each point-spectrum corresponded to an average over 20 scans of $1000 \mu \mathrm{m}$ for
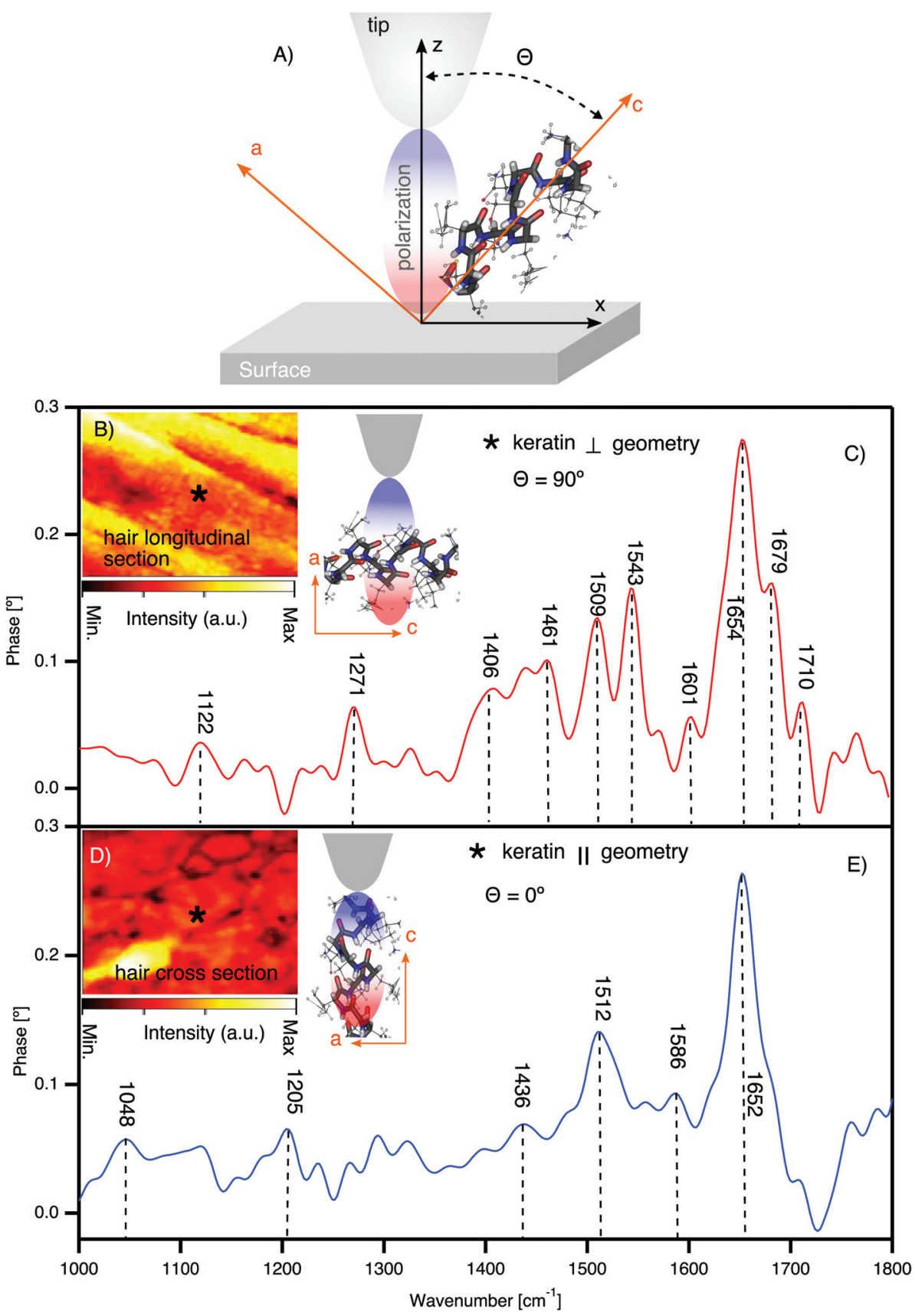

Fig. 3 Direct observation of keratin chemical bands and texture in the cortex of white sheep hair using synchrotron nano-FTIR. (A) The schematic of tip axis orientations with respect to the crystal substrate; $\theta$ indicating the angle between the $z$-axis (effective polarization) of the tip and the axis of the molecular dipole of the crystal c-axis. (B) IR broadband image of the hair longitudinal section with illustration of the molecular orientation with respect to tip polarization. $\theta=90^{\circ}$ means the effective polarization and the IR molecular dipole are orthogonal $(\perp){ }^{*}$ shows one of the positions where the spectra are acquired. (C) Nano-FTIR spectra of the keratin in the $\perp$ geometry. (D) IR broadband image of the hair cross-section with the illustration of the molecular orientation with respect to tip polarization. $\theta=0^{\circ}$ means the effective polarization and the IR molecular dipole are parallel $(\|) .{ }^{*}$ shows one of the positions where the spectra are acquired. (E) Nano-FTIR spectra of the keratin in the $\|$ geometry (detailed figures are shown in the ESI†). 
the optical path difference (OPD) which yield $10 \mathrm{~cm}^{-1}$ spectral resolution. For each scan, OPD was divided into 2048 pixels with $20 \mathrm{~ms}$ integration time per pixel.

\subsection{Nano-FTIR crystallographic vibrational anisotropy}

Recently it was demonstrated by Muller et al. ${ }^{50}$ that it is possible to determine molecular ordering by optical nano-crystallography using scattering-type scanning near-field optical microscopy. By applying the same method, here we address the orientation of keratin using nano-FTIR. In Fig. 3A the schematic of the relative orientation of the tip and molecular frames in the nano-FTIR measurements is presented. The optical near-field at the tip apex builds up an effective polarization parallel to the axis $(z)$. In this picture, $\theta$ indicates the angle between the effective polarization and the axis of molecular dipole. Hence, the relative orientation between the molecule and the tip can modulate the nano-FTIR signal: when the molecular dipole is parallel $\left(\theta=0^{\circ}\right)$ to the effective polarization, the IR signal is enhanced to the maximum; when the molecular dipole is orthogonal $\left(\theta=90^{\circ}\right)$ to the effective polarization, the IR signal is minimum. The anisotropic material response is proportional to the projection of the vibrational transition dipole.

\subsection{STEM measurements}

STEM imaging was performed with a scanning electron microscope "FEI Helios Nanolab 660", at LNNano Laboratory. STEM images were obtained on the cross and longitudinal sections of the hair (100 nm thickness) without any staining or coatings. The samples were sliced as described previously by using an ultramicrotome and transferred onto a TEM grid.

\section{Results and discussion}

We perform the in situ analysis of virgin black and white sheep hair to measure the spectro-vibrational response of native melanosomes, without any chemical treatment of the hair that could damage or modify the melanosomes. By using the novel Fourier transform infrared nanospectroscopy, ${ }^{51}$ it was possible to study and determine in situ the chemical fingerprint of the hair regions, with an unprecedented spatial resolution of $25 \mathrm{~nm}$. In Fig. 1 the nano-FTIR setup is shown illustrating nano-FTIR measurements on the virgin black and white sheep hairs.

To differentiate the complex morphology of the cortex of the black and white sheep hair, STEM analyses were performed on different slices of the hair samples. The results are shown for the cross-section, in Fig. 2A and B, and for the longitudinal cuts, in Fig. 2C and D.

The evident morphological contrast between two virgin hairs is shown, indicating unambiguously that melanosomes are present only in black sheep hair (see Fig. 2A and C). Virgin white sheep hair (see Fig. 2B and D) does not contain any pigment and this has been reported previously. ${ }^{9}$ From Fig. $2 \mathrm{~A}$ and $\mathrm{C}$ we note the granular clusters of melanosomes, and that they had an elliptical shape with an aspect ratio of about $2.5: 1$. Taking in account the poly-dispersion as one of the properties of the sheep's melanosomes, the average size is approximately: $250 \mathrm{~nm}$ (shorter diameter) $\times 800 \mathrm{~nm}$ (longer diameter).

\subsection{Keratin}

To elucidate the spectroscopic differences between pheomelanin and eumelanin, we measured virgin white sheep hair as a control for black hair. Notably (see Fig. 2B and D), white sheep hair does not contain melanosomes, and so it was possible to isolate the native keratin FTIR spectra (see Fig. 3). The results are summarized in Table 1.

Fig. 3 shows the nano-FTIR fingerprint of keratin obtained from the cortex region of white sheep hair. Nano-FTIR spectra were averaged over several points collected from different positions in slices cut longitudinally to the hair's axis (see Fig. 3B, the AFM tip axis is $\perp$ to the keratin IFs) and cross-section slices (see Fig. 3D, the AFM tip axis is $\|$ to the keratin IFs). Our $\mathrm{X}$-ray nano-diffraction results ${ }^{8}$ demonstrate that keratin has a

Table 1 Synchrotron nano-FTIR bands for sheep black and white hair obtained from the cortex region, shown in Fig. 3 and 4. The identification and assignments of the peaks were performed according to the published data ${ }^{51,52}$

\begin{tabular}{|c|c|c|c|}
\hline $\begin{array}{l}\text { Hair } \\
\text { origin }\end{array}$ & Color & $\begin{array}{l}\text { Wave number } \\
{\left[\mathrm{cm}^{-1}\right]}\end{array}$ & Assignments \\
\hline Sheep & White & $\begin{array}{l}\text { Keratin } \perp \text { geometry } \\
1122 \\
1271 \\
1406 \\
1461 \\
1509 \\
1543 \\
1601 \\
1654 \\
1679 \\
1710\end{array}$ & $\begin{array}{l}(\mathrm{S}=\mathrm{O}) \text {; cysteic dioxide } \\
\text { Amide III; } \beta \text {-sheet } \\
\text { Amide III } \\
\text { Amide II } \\
\text { Amide II } \\
\text { Amide II } \\
\text { Amide I; } \beta \text {-turn } \\
\text { Amide I; } \alpha \text {-helix } \\
\text { Amide I; } \beta \text {-sheet; random coil } \\
\text {-COOH }\end{array}$ \\
\hline Sheep & White & $\begin{array}{l}\text { Keratin } \| \text { geometry } \\
1048 \\
1205 \\
1436 \\
1512 \\
1586 \\
1652\end{array}$ & $\begin{array}{l}\mathrm{S}=\mathrm{O} ; \text { cysteic monoxide } \\
\text { Amide III } \\
\text { Amide II } \\
\text { Amide II } \\
\text { Amide II; } \beta \text {-turn } \\
\text { Amide I; } \alpha \text {-helix }\end{array}$ \\
\hline Sheep & Black & $\begin{array}{l}\text { Eumelanin } \\
1123 \\
1276 \\
1310 \\
1422 \\
1467 \\
1540 \\
1575 \\
1606 \\
1640\end{array}$ & $\begin{array}{l}(\mathrm{S}-\mathrm{O}) ; \text { cysteic dioxide } \\
(\mathrm{COH}) ; \text { phenolic stretching } \\
\mathrm{CN} \\
\delta(\mathrm{NH}), \delta(\mathrm{CH}), \nu(\mathrm{CN}) \\
\nu_{\text {ring }}, \nu(\mathrm{CN}), \delta(\mathrm{NH}), \delta(\mathrm{OH}) \\
\nu_{\text {ring }}, \delta(\mathrm{NH}), \delta(\mathrm{CH}) \\
\nu_{\text {ring }}, \text { indole NâH bending } \\
\nu(\mathrm{C}=\mathrm{O}) \\
\mathrm{C}=\mathrm{C}, \mathrm{C}=\mathrm{O}, \text { and/or COO- } \\
\text { stretching in the aromatic } \\
\text { cycle } \\
-\mathrm{COOH}\end{array}$ \\
\hline Sheep & Black & $\begin{array}{l}\text { Pheomelanin } \\
1124 \\
1172 \\
1213 \\
1272\end{array}$ & $\begin{array}{l}\text { (S-O); cysteic dioxide } \\
(\mathrm{S}-\mathrm{O}) \text {; cysteic acid } \\
(\mathrm{S}-\mathrm{O}) \\
(\mathrm{COH}) \text {; phenolic stretching }\end{array}$ \\
\hline
\end{tabular}


preferential, anisotropic behavior and the discovery of the keratin polarization effect (see Fig. $3 \mathrm{C}$ and E) is just an additional confirmation of such behavior.

There are mainly 4 characteristic IR bands in the white sheep hair nano-FTIR spectrum: (1) band related to S-O stretching around $1122 \mathrm{~cm}^{-1}$, (2) IR bands of amide III, from $1200 \mathrm{~cm}^{-1}$ to $1300 \mathrm{~cm}^{-1}$, (3) amide II bands, from $1400 \mathrm{~cm}^{-1}$ to $1600 \mathrm{~cm}^{-1}$ and (4) bands associated with the stretching vibrations of amide I, from $1600 \mathrm{~cm}^{-1}$ to $1700 \mathrm{~cm}^{-1}$. We attribute the relative intensity of these peaks to the orientation of the IFs with respect to the polarization of the optical-near field parallel to the tip axis ${ }^{50,53}$ (see Fig. 3C and E). As recently demonstrated by Muller et al. (2016), ${ }^{50}$ the distribution of the molecular orientation and disorder can be determined by correlating the polarization of the exciting optical near-field with the direction of the infrared dipole of molecular groups on the sample. Nevertheless, hair has a complex and heterogeneous structure at the nanometer length scale, ${ }^{8}$ and the randomness should be considered during such measurements of molecular orientation and crystallinity.

The prominent amide I region in Fig. 3C is split into four peaks, where the most intense peak of around $1654 \mathrm{~cm}^{-1}$ is characteristic of $\alpha$-helix, the peak of around $1679 \mathrm{~cm}^{-1}$ is related to $\beta$-sheet, the peak at $1601 \mathrm{~cm}^{-1}$ is related to $\beta$-turn and the peak at $1710 \mathrm{~cm}^{-1}$ is assigned to the $-\mathrm{COOH}$ stretching. ${ }^{54,55}$ However, we have to point out that in our spectra, the frequency around $1672 \mathrm{~cm}^{-1}$, typical of beta turn, ${ }^{54}$ is not resolved. It may be hidden between the peaks at $1654 \mathrm{~cm}^{-1}$ and $1679 \mathrm{~cm}^{-1}$, which are most pronounced. The transformation from $\alpha$-helix to $\beta$-pleated structure can occur during the breakage of the disulfide bonds ( $\mathrm{S}-\mathrm{S}$ bonds). Such breakage can be induced in different ways, for example: sunlight, weathering, stretching or by application of chemical products to the hair. For example, the formation of cysteic acid, which is one of the possible by-products of S-S breakage, has been reported previously. ${ }^{56-58}$ The excessive de-colorization of melanin as well as the oxidation of cysteine, which is a prime constituent amino acid of keratin, may create the by-product of cysteic acid. However, the localization of cysteic acid and the spatial distribution inside the hair were not clarified until now. Furthermore, it has been shown in untreated hair ${ }^{58}$ that the photo-oxidation of the hair can produce minor amounts of cysteic acid as well as traces of cysteine- $S$-monoxide as well as cysteine-S-dioxide. ${ }^{57,59}$ In our case we do exclude chemical and

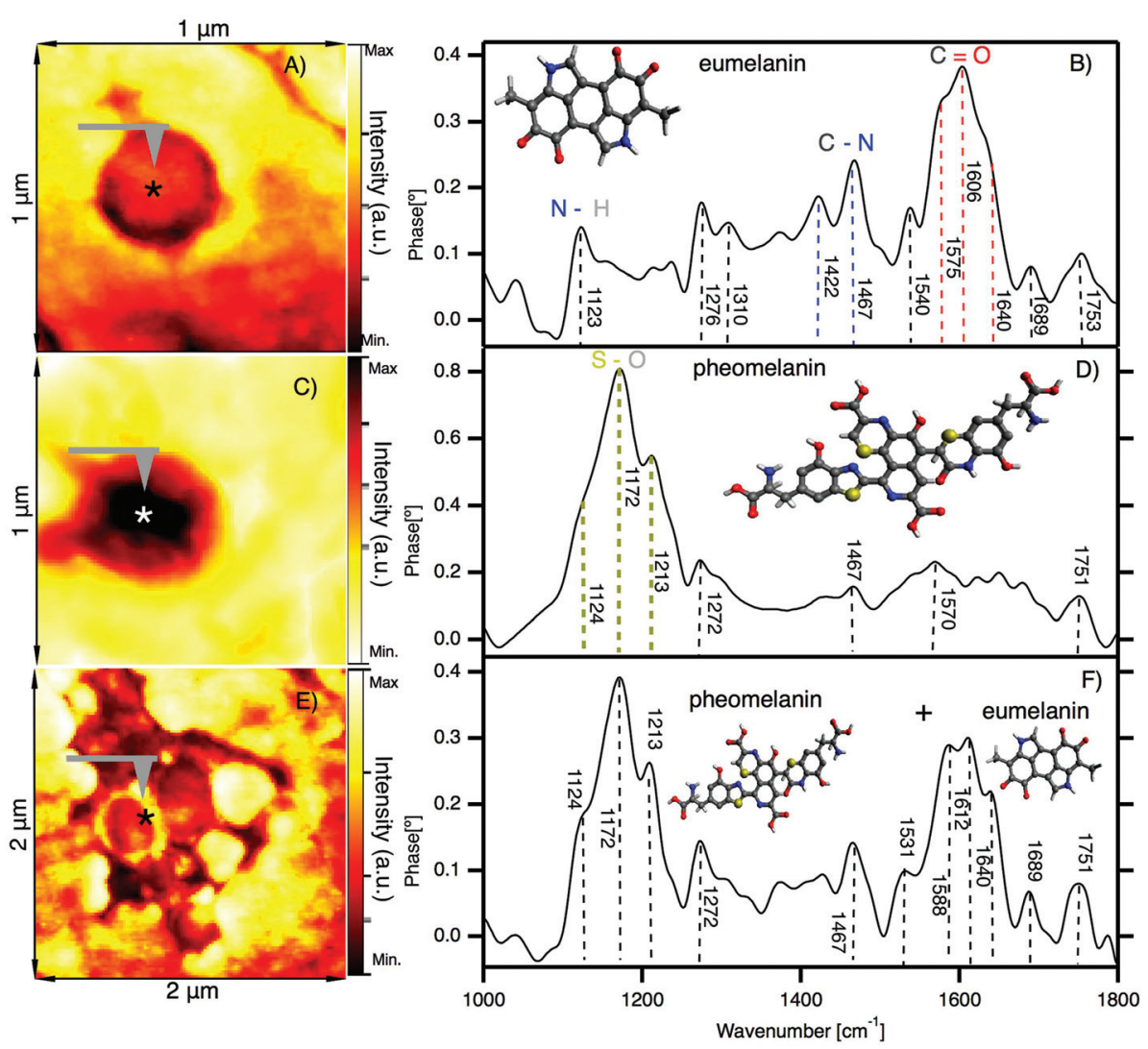

Fig. 4 In situ observation of melanosomes in the cortex of black sheep hair by nano-FTIR. (A) IR broadband image of a melanosome showing the position (*) where the spectra are acquired. (B) Nano-FTIR spectra of eumelanin; inset: schematic representation of the eumelanin partial chemical formula. (C) IR broadband image of a melanosome showing the position (*) where the spectra are acquired. (D) Nano-FTIR spectra of pheomelanin; inset: schematic representation of the pheomelanin partial chemical formula. (E) IR broadband image of clusters of melanosomes showing the position $(*)$ where the spectra are acquired. (F) Mixed nano-FTIR signal of eumelanin and pheomelanin; inset: schematic representation of the eumelanin and pheomelanin chemical formula (detailed figures are shown in the ESI $\dagger$ ). 
stretching factors, since such treatments were not applied and considering the low intensity of the peak at $1122 \mathrm{~cm}^{-1}$ of S-O stretching, it indicates that the oxidation process is reduced.

\subsection{Eumelanin and pheomelanin}

In Fig. 4 we show the results of nano-FTIR in situ measurements of two characteristic melanosomes of sheep black hair: pheomelanin and eumelanin.

Fig. 4A shows the first native eumelanin FTIR spectra obtained directly from the virgin black sheep hair without any chemical manipulation or extraction. The inset shows the infrared near-field image of a melanosome in the cortex region of black sheep hair with the schematic chemical formula of eumelanin. The majority (95\%) of the melanosomes investigated in the black sheep hair contains eumelanin. However, the very small amount of the melanosomes present the characteristic pheomelanin oxidation state (see Fig. 4B) or sometimes it is a combination of the two FTIR spectra found in the cluster regions of melanosomes (see Fig. 4C), shown also by ref. 60.

We note that there is a compelling difference in the nanoFTIR spectra of pheomelanin and eumelanin. The region from $1000 \mathrm{~cm}^{-1}$ to $1300 \mathrm{~cm}^{-1}$ can be characteristic only for pheomelanin with extremely strong peaks at $1124 \mathrm{~cm}^{-1}, 1172 \mathrm{~cm}^{-1}$ and $1213 \mathrm{~cm}^{-1}$ which are given by $\mathrm{S}-\mathrm{O}$ symmetric stretching vibration, generated by the oxidation of sulfonic groups present in pheomelanin. On the contrary, for eumelanin we found the characteristic IR bands from 1400 to $1770 \mathrm{~cm}^{-1}$ with peaks clearly resolved at $1422 \mathrm{~cm}^{-1}, 1467 \mathrm{~cm}^{-1}, 1540 \mathrm{~cm}^{-1}$ and $1572 \mathrm{~cm}^{-1}$, which are unique for eumelanin.

To further discuss the differences between eumelanin and pheomelanin we have to point out that hair is rich in elements such as: $\mathrm{Ca}, \mathrm{Cu}, \mathrm{Fe}$ and $\mathrm{Zn}$, but it is still unclear how exactly the elements are distributed in the hair. The recent results by Edwards, N. P. et al. (2016), ${ }^{61}$ from studies in bird feathers, show that the elemental distribution is controlled mainly by melanin. They found that the key difference between eumelanin and pheomelanin is the metal bonding. For example, in pheomelanin, $\mathrm{Zn}$ is bonded to $\mathrm{S}$, as compared with the $\mathrm{Zn}-$ $\mathrm{N} / \mathrm{O}$ in eumelanin. Studies on eumelanin have found metals to be predominantly bound to oxygen either in carboxyl groups, or in hydroxyl groups. We note that white sheep hair also contains the same elements, but in smaller quantities compared to black sheep hair; most probably these elements are bound to keratin, considering that white sheep hair does not contain melanosomes. Our nano-FTIR data do address the complexity due to these elements (concentration and bonding of these elements). So, it is unclear if such elements are involved in the oxidation processes of the hair and if they are detectable by IR, but we should not exclude the possible presence of $\mathrm{Zn}$ sulfate in pheomelanin. ${ }^{61,62}$

This is the direct in situ distinction of the chemical fingerprint of two melanosomes, performed without any chemical labeling. With this finding we can demonstrate that nano-FTIR is a non-destructive technique capable to detect and spatially resolve the chemical heterogeneity of hair.

\section{Conclusion}

We have revealed and spatially resolved the native chemical fingerprint of keratin as well as melanosomes without altering the hair chemistry. Furthermore, by measuring black and white sheep hair, we have identified the characteristic IR molecular vibrational fingerprints of two types of melanosomes, respectively: eumelanin and pheomelanin. This study demonstrates that nano-FTIR can be used to spatially resolve the chemical and oxidation processes of hair components at the nano-scale resolution. Moreover, our findings add an important understanding to the nature of hair color as well as to the comprehension on the roles of melanosomes in different biological processes.

\section{Conflicts of interest}

There are no conflicts to declare.

\section{Acknowledgements}

The LNLS and LNNano were supported by the The Ministry of Science, Technology, Innovation and Communication of Brazil (MCTIC). The authors acknowledge the LNLS for providing the beamtime.

\section{References}

1 R. D. B. Fraser, G. E. Rogers, B. K. Filshie and T. P. MacRae, J. Mol. Biol., 1963, 7, 90-91.

2 L. Pauling and R. B. Corey, Proc. Natl. Acad. Sci. U. S. A., 1951, 37, 261.

3 C. Merigoux, F. Briki, F. Sarrot-Reynauld, M. Salome, B. Fayard, J. Susini and J. Doucet, Biochim. Biophys. Acta, 2003, 1619, 53.

4 M. Wade, I. Tucker, P. Cunningham, R. Skinner, F. Bell, T. Lyons, K. Patten, L. Gonzalez and T. Wess, Int. J. Cosmet. Sci., 2013, 35, 430.

5 D. P. Harland, R. J. Walls, J. A. Vernon, J. M. Dyer, J. L. Woods and F. Bell, J. Struct. Biol., 2014, 185, 397.

6 D. A. Parry, J. Struct. Biol., 2014, 186, 320.

7 A. Iida and T. Noma, Nucl. Instrum. Methods Phys. Res., Sect. $B, 1993,82,129-138$.

8 V. Stanić, J. Bettini, F. E. Montoro, A. Stein and K. EvansLutterodt, Sci. Rep., 2015, 5, 17347.

9 A. Spätha, M. Meyera, S. Semmlera and R. H. Finka, Micron, 2015, 70, 34-40.

10 J. A. Clarke, D. T. Ksepka, R. Salas-Gismondi, A. J. Altamirano, M. D. Shawkey, L. D'Alba, J. Vinther, T. J. DeVries and P. Baby, Science, 2010, 330, 954-956.

11 L. D'Alba, V. Saranathan, J. A. Clarke, J. A. Vinther, R. O. Prum and M. D. Shawkey, Biol. Lett., 2011, 7, 543-546. 
12 V. Saranathan, C. O. Osuji, S. G. J. Mochrie, H. Noh, S. Narayanan, A. Sandy, E. R. Dufresne and R. O. Prum, Proc. Natl. Acad. Sci. U. S. A., 2010, 107, 11676-11681.

13 S. V. Saenko, J. Teyssier, D. van der Marel and M. C. Milinkovitch, BMC Biol., 2013, 11, 105.

14 G. F. Grether, G. R. Kolluru and K. Nersissian, Biol. Rev. Cambridge Philos. Soc., 2004, 79, 583-610.

15 J. Teyssier, S. V. Saenko, D. van der Marel and M. C. Milinkovitch, Nat. Commun., 2015, 6, 6368.

16 S. Chadwick, R. Heath and M. Shah, Indian J. Plast. Surg., 2012, 45, 403-411.

17 L. Pelkonen, M. Reinisalo, E. Morin-Picardat, H. Kidron and A. Urtti, PLoS One, 2016, 1-13.

18 M. Cichorek, M. Wachulska, A. Stasiewicz and A. Tymińska, Postep. Derm. Alergol., 2013, XXX, 30-41.

19 J. Borovansk and P. Hach, Arch. Dermatol. Res., 1986, 279, 54-58.

20 T. Gorniak, T. Haraszti, V. M. Garamus, A. R. Buck, T. Senkbeil, M. Priebe, A. Hedberg-Buenz, D. Koehn, T. Salditt, M. Grunze, M. G. Anderson and A. Rosenhahn, PLoS One, 2014, 9, e90884.

21 L. M. Miller and P. Dumas, Biochim. Biophys. Acta, 2006, 1758, 846-857.

22 M. C. Martin, C. Dabat-Blondeau, M. Unger, J. Sedlmair, D. Y. Parkinson, H. A. Bechtel, B. Illman, J. M. Castro, M. Keiluweit, D. Buschke, B. Ogle, M. J. Nasse and C. J. Hirschmugl, Nat. Methods, 2013, 10, 861-864.

23 S. Kawata and Y. Inouye, Ultramicroscopy, 1995, 57, 313-317.

24 F. Zenhausern, Y. Martin and H. K. Wickramasinghe, Science, 1995, 269, 1083-1085.

25 B. Knoll and F. Keilmann, Nature, 1999, 399, 134-137.

26 F. Huth, M. Schnell, J. Wittborn, N. Ocelic and R. Hillenbrand, Nat. Mater., 2011, 10, 352-356.

27 P. Hermann, A. Hoehl, P. Patoka, F. Huth, E. Rühl and G. Ulm, Opt. Express, 2013, 21, 2913-2919.

28 P. Hermann, A. Hoehl, G. Ulrich, C. Fleischmann, A. Hermelink, B. Kästner, P. Patoka, A. Hornemann, B. Beckhoff, E. Rühl and G. Ulm, Opt. Express, 2014, 22, 17948-17958.

29 H. A. Bechtel, E. A. Muller, R. L. Olmon, M. C. Martin and M. B. Raschke, Proc. Natl. Acad. Sci. U. S. A., 2014, 111, 7191-7196.

30 B. Pollard, F. C. B. Maia, M. B. Raschke and R. O. Freitas, Nano Lett., 2016, 16, 55-61.

31 R. O. Freitas, C. Deneke, F. C. B. Maia, H. G. Medeiros, T. Moreno, P. Dumas, Y. Petroff and H. Westfahl, Opt. Express, 2018, 26, 11238-11249.

32 F. Huth, A. Govyadinov, S. Amarie, W. Nuansing, F. Keilmann and R. Hillenbrand, Nano Lett., 2012, 12, 3973-3978.

33 B. Pollard, E. A. Muller, K. Hinrichs and M. B. Raschke, Nat. Commun., 2014, 5, 3587.

34 I. Amenabar, S. Poly, W. Nuansing, E. H. Hubrich, A. A. Govyadinov, F. Huth, R. Krutokhvostov, L. Zhang, M. Knez, J. Heberle, A. M. Bittner and R. Hillenbrand, Nat. Commun., 2013, 4, 2890.
35 S. Amarie, P. Zaslansky, Y. Kajihara, E. Griesshaber, W. W. Schmahl and F. Keilmann, Beilstein J. Nanotechnol., 2012, 3, 312-323.

36 I. D. Barcelos, A. R. Cadore, L. C. Campos, A. Malachias, K. Watanabe, T. Taniguchi, F. C. B. Maia, R. Freitas and C. Deneke, Nanoscale, 2015, 7, 11620-11625.

37 Z. Shi, H. A. Bechtel, S. Berweger, Y. Sun, B. Zeng, C. Jin, H. Chang, M. C. Martin, M. B. Raschke and F. Wang, ACS Photonics, 2015, 2, 790-796.

38 P. Patoka, G. Ulrich, A. E. Nguyen, L. Bartels, P. A. Dowben, V. Turkowski, T. S. Rahman, P. Hermann, B. Kästner, A. Hoehl, G. Ulm and E. Rühl, Opt. Express, 2016, 24, 11541164.

39 D. Grasseschi, D. A. Bahamon, F. C. B. Maia, A. H. C. Neto, R. O. Freitas and C. J. S. de Matos, 2D Mater., 2017, 4, 035028.

40 I. D. Barcelos, A. R. Cadore, A. B. Alencar, F. C. B. Maia, E. Mania, R. F. Oliveira, C. C. B. Bufon, Â. Malachias, R. O. Freitas, R. L. Moreira and H. Chacham, ACS Photonics, 2018, 5, 1912-1918.

41 A. Dazzi, R. Prazeres, F. Glotin and J. M. Ortega, Opt. Lett., 2005, 30, 2388-2390.

42 A. Dazzi, F. Glotin and R. Carminati, J. Appl. Phys., 2010, 107, 124519.

43 J. R. Felts, K. Kjoller, C. B. Prater and W. P. King, 2010 IEEE 23rd International Conference on Micro Electro Mechanical Systems (MEMS), 2010, pp. 136-139.

44 J. Houel, E. Homeyer, S. Sauvage, P. Boucaud, A. Dazzi, R. Prazeres and J.-M. Ortéga, Opt. Express, 2009, 17, 1088710894.

45 F. Lu, M. Jin and M. A. Belkin, Nat. Photonics, 2014, 8, 307312.

46 L. Wang, H. Wang, M. Wagner, Y. Yan, D. S. Jakob and X. G. Xu, Sci. Adv., 2017, 3, e1700255.

47 P. M. Donaldson, C. S. Kelley, M. D. Frogley, J. Filik, K. Wehbe and G. Cinque, Opt. Express, 2016, 24, 1852-1864.

48 L. Tetard, A. Passian, R. H. Farahi, T. Thundat and B. H. Davison, Nat. Nanotechnol., 2015, 10, 870-877.

49 F. Keilmann and R. Hillenbrand, Philos. Trans. R. Soc., A, 2004, 362, 787-805.

50 E. A. Muller, B. Pollard, H. A. Bechtel, P. van Blerkom and M. B. Raschke, Sci. Adv., 2016, 2, 1-6.

51 I. Amenabar, S. Poly, M. Goikoetxea, W. Nuansing, P. Lasch and R. Hillenbrand, Nat. Commun., 2017, 8, 14402.

52 M. G. Bridelli and P. R. Crippa, J. Phys. Chem. B, 2010, 114, 9381-9390.

53 T. Ishibashi and Y. Cai, Nanoscale Res. Lett., 2015, 10, 1-8.

54 A. Barth, Biochim. Biophys. Acta, 2007, 1767, 1073-1101.

55 A. J. Zhou, H. L. Liu and Z. Q. Du, Skin. Res. Technol., 2015, 21, 119-128.

56 K. Watanabe, K. Nagami, K. Suzuta, T. Maeda and L. Ito, Adv. Life Sci., 2015, 5, 85-89.

57 D. Ammann, R. Becker, A. Kohl, J. Hanisch and I. Nehls, Forensic Sci. Int., 2014, 244, 30-35.

58 K. Bito, M. Okuno, H. Kano, S. Tokuhara, S. Naito, Y. Masukawa, P. Leproux, V. Couderc and H. Hamaguchi, J. Phys. Chem. B, 2012, 116, 1452-1457. 
59 S. Hilterhausbong and H. Zahn, Int. J. Cosmet. Sci., 1987, 9, 101-109.

60 C. Colleary, A. Dolocan, J. Gardner, S. Singh, M. Wuttke, R. Rabenstein, J. Habersetzer, S. Schaal, M. Feseha, M. Clemens, B. Jacobs, E. Currano, L. Jacobs, R. Sylvestersen, S. Gabbott and J. Vinther, Proc. Natl. Acad. Sci. U. S. A., 2015, 112, 12592-12597.
61 N. P. Edwards, A. van Veelen, J. Anné, P. L. Manning, U. Bergmann, W. I. Sellers, V. M. Egerton, D. Sokaras, R. Alonso-Mori, K. Wakamatsu, S. Ito and R. A. Wogelius, Sci. Rep., 2016, 6, 34002.

62 H. E. Barden, U. Bergmann, N. P. Edwards, V. M. Egerton, P. L. Manning, S. Perry, A. van Veelen, R. A. Wogelius and B. E. van Dongen, Palaeobio. Palaeoenv., 2015, 95, 33-45. 